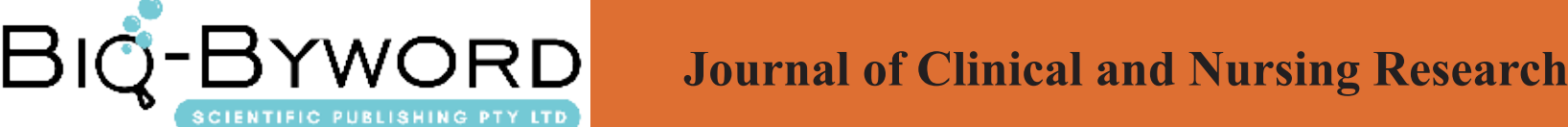

\section{Efficacy and safety of rhubarb aconite decoction combined with acupoint application in the treatment of chronic renal failure (CRF)}

\author{
LI Yang ${ }^{1}$ FU Ning ${ }^{2 *}$ ZHANG Wei ${ }^{2}$ XIE Jun ${ }^{2}$
}

1. Hanzhong People's Hospital, Hanzhong, Shaanxi Province, 723000

\section{ARTICLE INFO}

Article history:

Published online: 15th July, 2017

Key words:

Rhubarb aconite acacia soup acupoint application

renal failure; efficacy

safety

*Corresponding Author

Fu Ning, Hanzhong City, Shaanxi Province, Hantai District, North District, No. 251, Hanzhong City People's Hospital. Email address: funing2018.163com.

\section{ABSTRACT}

Objective To study the efficacy and safety of rhubarb aconite decoction combined with acupoint application in the treatment of chronic renal failure (CRF). Methods 106 cases of CRF patients were randomly divided into two groups, the control group of 53 cases of conventional Western medicine treatment, the treatment group of 53 cases of rhubarb aconite combined with acupoint application, 2 groups were treated continuously for 20 d. (UA), blood urea nitrogen (BUN) and endogenous creatinine clearance (Ccr) were measured before and after treatment. The levels of serum creatinine $(\mathrm{Cr})$, serum creatinine (UA), blood urea nitrogen (BUN) and endogenous creatinine clearance (Ccr) were measured and compared. Level, and observe the occurrence of adverse reactions during the two groups of treatment. Results The total effective rate of the treatment group was significantly higher than that of the control group ( $\mathrm{P}$ 0.05). The scores of TCM group were significantly lower than those of the control group $(\mathrm{P}<0.05) \mathrm{BUN}, \mathrm{Ccr}$ renal function were significantly improved, and the treatment group was significantly higher than the control group $(\mathrm{P}<0.05)$. There was no significant difference in the incidence of adverse reactions between the two groups $(\mathrm{P}>0.05)$. Conclusion Treatment of CRF with rhubarb aconite and acupoint application can effectively improve the renal function of patients, the clinical curative effect is remarkable, the adverse reaction is small, and it is worthy to be popularized. 


\section{Introduction}

Chronic renal failure (CRF) ${ }^{[1]}$ belongs to a variety of kidney disease in a late clinical syndrome, mainly for water and electrolyte disorders, metabolites and toxins retention, acid-base balance disorders, the systemic involvement of the system, etc. The CRF course of disease, seriously affecting the quality of life of patients, has become an important issue threatening public health and health. The current clinical use of CRF late dialysis and kidney transplantation methods ${ }^{[2]}$, high cost, resulting in economic burden for patients and their families cannot afford. Therefore, active exploration of non-dialysis and surgical approach to the treatment of early and mid-term CRF significant. Studies have shown that rational Chinese medicine therapy can effectively delay the progress of renal dysfunction, with good advantages and characteristics ${ }^{[3]}$. In this study, 53 patients with CRF were treated with rhubarb aconite and acupoints combined with acupoints and compared with their clinical efficacy and safety. The aim was to provide a reference for guiding clinical medicine. It is reported as follows:

\section{Clinical data}

1.1 General information On our hospital in August 2014 to February 2016 treatment of CRF patients in 106 cases are in line with "Practical Internal Medicine" ${ }^{[4]}$ CRF diagnostic criteria. Chinese medicine diagnostic criteria refer to the "traditional Chinese medicine clinical guidelines" ${ }^{[5]}$, the main symptoms: eat less appetite, aversion to cold; secondary disease: fatigue fatigue, wrist abdominal fullness, nausea and / or vomiting, Yaoxisuanruan, Heavy, nocturia long, mouth sticky; tongue and pulse: tongue partial fat, pale tongue, greasy greasy fur, pulse slippery or Shen fine. With 2 main symptoms, 3 times the disease, combined with tongue and pulse, can be diagnosed as cloud poison intrinsic, spleen and kidney yang deficiency. Inclusion criteria: Meet the clinical stage of CRF patients with clinical criteria ${ }^{[4]}$; No serious complications; Age 18 to 70 years; All patients are willing to sign informed consent. Exclusion criteria: Patients with acute renal failure; Before or during treatment using hemodialysis patients; Accompanied by liver fibrosis or chronic liver disease patients; Recent serious infection, acidosis, water and electrolyte disorders ; On this study of drug allergy patients. The subjects in this group were divided into two groups: the control group $(\mathrm{n}=28)$ and the female $(n=25)$ had the mean age of 34-67 $(48.52 \pm$ 9.72) years. The course of the course was 9-36 (25.18 \pm 4.57 ) months Clinical stage: 26 cases of stage $\square, 27$ cases of stage $\square$; 18 cases of chronic glomerulonephri- tis, 14 cases of diabetic nephropathy, 21 cases of gouty kidney disease. There were 31 males and 22 females in the treatment group. The age was $32-68(47.13 \pm 10.06)$ years; the course of disease was 11-39 $(26.54 \pm 5.14)$ months. The clinical stage was 29 cases in stage $\square$ and 24 cases in stage $\square$ Glomerulonephritis in 16 cases, 18 cases of diabetic nephropathy, 19 cases of gouty kidney disease. There was no significant difference in gender, age, course of disease and clinical stage between the two groups $(\mathrm{P}>0.05)$.

1.2 Treatment group 2 were low protein, low salt, low phosphorus diet, given blood pressure, diuretic and anti-infection and other conventional treatment, timely correction of acid-base balance disorders and water and electrolyte disorders. The control group received oral administration of ferulic acid piperazine tablets (Kangpu Pharmaceutical Co., Ltd., Baoshengkang, Chinese medicine quasi-word H43021760, $50 \mathrm{mg}$ / tablet) 150 $\mathrm{mg}$, coenzyme Q10 capsules (Shanghai Xinyi Pharmaceutical Co., Ltd., Xinyi, Chinese medicine quasi-word H19999132, $10 \mathrm{mg} /$ tablets) $20 \mathrm{mg}$ treatment, 3 times a day. Treatment group to rhubarb aconite Asarum soup combined with acupoint application. Rhubarb aconite prescription: rhubarb, the system of aconite, salvia, raw Astragalus, Poria $30 \mathrm{~g}$, Alisma, Atractylodes $10 \mathrm{~g}$, Asarum $3 \mathrm{~g}$. Usage and Dosage: The above prescription decoction filter, take the supernatant $300 \mathrm{~mL}, 1$ day, sooner or later each time, warm water delivery service. Acupoints prescription: raw rhubarb, raw Chuanxiong, raw Gan Sui the $30 \mathrm{~g}$, raw euphorbia $20 \mathrm{~g}$, incense, borneol $10 \mathrm{~g}$ each. The above drugs to study the first fine, and then add the appropriate amount of vinegar which stir evenly, and finally the mixture into the gauze bag. Alternating in the patient Shenque, acupoints Shenshu (bilateral) 2 points, each $2 \mathrm{~h}, 1$ day, each time to control the gauze bag outside the temperature. 2 groups of treatment were $20 \mathrm{~d}$.

\subsection{Observe indicators}

(1) Clinical observation. Record the clinical efficacy of two groups of patients. According to the "Chinese medicine new drug clinical research guidelines" ${ }^{[5]}$ in the standard of the efficacy of chronic renal failure assessment: markedly: clinical symptoms improved significantly, TCM syndrome score decreased by $\geq 60 \%$, $\mathrm{Cr} \geq 20 \%$ or Ccr increased $\geq 20 \%$; effective : Clinical symptoms improved, Chinese medicine syndrome score $\geq 30 \%$, Cr reduction $\geq 10 \%$ or Ccr increased $\geq$ $10 \%$; stability: clinical symptoms improved significantly, $\mathrm{Cr}<10 \%$, or no increase; invalid: no improvement in clinical symptoms Or aggravated, no change or rise in $\mathrm{Cr}$. The total effective rate is effective, the effective 
Table 12 groups of patients with clinical efficacy comparison results [n (\%)]

\begin{tabular}{lllllll}
\hline Group & Cases & $\begin{array}{l}\text { Remarkably } \\
\text { effective }\end{array}$ & Effective & Stable & Invalid & Efficacy \\
\hline $\begin{array}{l}\text { Contro } 153 \\
\text { group }\end{array}$ & 21(39.62) & $18(33.96)$ & $9(16.98)$ & $5(9.43)$ & $39(73.58)$ \\
$\begin{array}{l}\text { Treatment } \\
\text { group }\end{array}$ & $33(62.26)$ & $16(30.19)$ & $3(5.66)$ & $1(1.89)$ & $49\left(92.45^{\mathrm{a}}\right)$ \\
\hline
\end{tabular}

Note: a: compared with the control group, $\mathrm{P}<0.05$.

proportion of the sum.

(2) TCM syndrome score. Comparison of two groups of patients before and after treatment of traditional Chinese medicine syndrome integral changes, the main symptoms: eat less appetite, aversion to cold, according to light, medium and heavy were given 2, 4, 6 points; Fatigue, wrist abdominal fullness, nausea and/or vomiting, relative weakness in the loins and knees, limb trapped heavy, nocturia long, mouth sticky, according to light, medium and heavy were given $1,2,3$ points, the total score.

(3) Indicators of renal function and adverse reactions. The levels of serum creatinine $(\mathrm{Cr})$, Blood uric acid (UA), Blood urea nitrogen (BUN), Endogenous creatinine clearance $(\mathrm{Ccr})$ and other changes in renal function.

(4) Adverse reactions. To monitor the two groups of patients during treatment of blood lipids, liver function, electrolyte and other indicators of changes in statistical dizziness, diarrhea, abdominal distension, constipation and other adverse reactions occur, calculate the incidence of adverse reactions.

1.4 Statistical Methods. SPSS 20.0 software was used to analyze the data of the experimental data, clinical efficacy, adverse reactions and other count data rate (\%) description, the use of chi-square test, TCM syndrome scores and renal function indicators and other measurement data are used The number of \pm standard deviation $(\bar{x} \pm s)$ description, the use of t-test for group or group comparison, with $\mathrm{P}<0.05$ for the difference was statistically significant.

\section{Results}

2.1 The total effective rate was $92.45 \%$ in the clinical treatment group, which was significantly higher than that in the control group $(73.58 \%, \mathrm{P}<0.05)$. See Table 1 for details.

There was no significant difference in TCM syndrome scores between the two groups before treatment $(\mathrm{P}>$ $0.05)$. The scores of TCM syndrome scores were significantly lower in the two groups after treatment, and the decrease of the treatment group was more significant than that of the control group $(\mathrm{P}<0.05)$. See Table 2 for details.

There was no significant difference in the levels of $\mathrm{Cr}$, UA, BUN and Ccr renal function between the two groups before treatment $(\mathrm{P}>0.05)$. After treatment, the above indexes were significantly improved, and the treatment group was significantly improved higher than the control group $(\mathrm{P}<0.05)$. See Table 3 for details.

2.4 Adverse reactions during the treatment group showed bloating in 2 cases, 2 cases of constipation,

Table 22 groups of patients before and after treatment of TCM syndrome score results $(\bar{x} \pm s, \%)$

\begin{tabular}{llll}
\hline Group & Cases & Before treatment & After treatment \\
\hline Control group & 53 & $16.35 \pm 4.61$ & $5.75 \pm 1.47^{\mathrm{a}}$ \\
Treatment group & 53 & $17.04 \pm 5.89$ & $3.06 \pm 0.84^{\mathrm{ab}}$ \\
\hline
\end{tabular}

Note: ${ }^{a}$ : compared with before treatment, $\mathrm{P}<0.05$, ${ }^{\mathrm{b}}$ : compared with the control group, $\mathrm{P}<0.05$. 
Table 3 Comparison of changes in renal function before and after treatment in 2 groups

\begin{tabular}{|c|c|c|c|c|c|}
\hline Group & Time & $\mathrm{Cr}(\mu \mathrm{mol} / \mathrm{L})$ & $\mathrm{UA}(\mu \mathrm{mol} / \mathrm{L})$ & $\mathrm{BUN}(\mathrm{mmol} / \mathrm{L})$ & $\operatorname{Ccr}(\mathrm{mL} / \mathrm{min})$ \\
\hline \multirow[t]{2}{*}{$\begin{array}{l}\text { Control } \\
\text { group }\end{array}$} & $\begin{array}{l}\mathrm{B} \text { e for e } \\
\text { treatment }\end{array}$ & $358.31 \pm 86.49$ & $493.56 \pm 96.34$ & $15.17 \pm 4.32$ & $27.45 \pm 7.10$ \\
\hline & $\begin{array}{l}\text { After treat- } \\
\text { ment }\end{array}$ & $326.75 \pm 63.34^{\mathrm{a}}$ & $376.28 \pm 76.48^{\mathrm{a}}$ & $13.63 \pm 3.09^{\mathrm{a}}$ & $31.16 \pm 9.73^{\mathrm{a}}$ \\
\hline \multirow{2}{*}{$\begin{array}{l}\text { T r e a t - } \\
\mathrm{m} \text { e } \mathrm{n} \mathrm{t} \\
\text { group }\end{array}$} & $\begin{array}{l}\mathrm{B} \text { e for e } \\
\text { treatment }\end{array}$ & $361.52 \pm 90.47$ & $498.27 \pm 87.69$ & $14.98 \pm 5.04$ & $27.62 \pm 6.97$ \\
\hline & $\begin{array}{l}\text { After treat- } \\
\text { ment }\end{array}$ & $304.28 \pm 53.49^{\mathrm{ab}}$ & $257.38 \pm 79.64^{\mathrm{ab}}$ & $11.87 \pm 2.67^{\mathrm{ab}}$ & $37.02 \pm 10.64^{\mathrm{ab}}$ \\
\hline
\end{tabular}

Note: ${ }^{\text {a: }}$ compared with before treatment, $\mathrm{P}<0.05,{ }^{\mathrm{b}}$ : compared with the control group, $\mathrm{P}<0.05$.

dizziness in 1 case, the incidence of adverse reactions was $9.43 \%$, the treatment group 1 case of diarrhea, dizziness, 1 case, the incidence of adverse reactions was $3.77 \%$ There was no significant difference in the incidence of adverse reactions between the two groups $(\mathrm{P}$ $>0.05)$.

\section{Discussion}

$\mathrm{CRF}$ is a variety of clinical kidney disease in patients with advanced diseases, easily lead to water and electrolyte disorders, renal dysfunction, resulting in toxins cannot be discharged in time, the final uremia ${ }^{[6]}$. At present, dialysis and renal transplantation in the treatment of CRF uremia has a certain effect, but the equipment requirements and treatment costs are high, it is difficult to widely use in clinical practice. And traditional Chinese medicine ${ }^{[7]}$ in the treatment of CRF can syndrome differentiation, learn from each other, the effect is significant. The motherland medicine believes that CRF belongs to the category of "edema", "customs", and "consumptive" and so on ${ }^{[8]}$. The basic pathogenesis ${ }^{[9]}$ for the wet poison intrinsic, spleen and kidney yang. "Doctors must read" said: "Kidney water is dirty, Yuan Yang apartment Yan. Life door fire bad, neither selfish cold, and cannot warm the spleen, the yin from the sun and refined into water, so Edema of the card, mostly fire also. "Spleen and kidney storage and health dysfunction, cannot distinguish between voicing, wet cloud stagnation, wet cloud drowning intrinsic, keep for a long time to become poisonous, congestion triple burner, causing rapid renal failure. So to take cold and heat, and attack and complement the method ${ }^{[10]}$ for treatment, both emptying the detoxification, but also spleen and kidney, both symptoms and symptoms. In this study, the author used rhubarb aconite acacia soup combined with the application of warm spleen and kidney.

Acupoints in the application of rhubarb ${ }^{[1]}$ can stasis through the pass; raw Chuanxiong can blood circulation; and raw Kansui good at removing swelling and bruises; raw fuzi benefits in the auxiliary fire Yang; Shen incense with the effect of remove gas and pain; Borneol can be soothing swelling and pain, scattered internal flame. The use of traditional Chinese medicine acupoints applied in the Shenshu points and Shenque points two points, the drug through the skin to penetrate the absorption, direct access to the patient root, the role of the meridian, play a pharmacodynamic effect.

Acupoint application ${ }^{[12]}$ not only can play strengthening the body and removing evil factors role, the role of two-way adjustment, but also both side effects, simple operation characteristics. Studies have shown that [13], acupoint application can improve the kidney and intestinal microcirculation, improve drug bioavailability, increase intestinal peristalsis and renal blood flow, reduce the drug in the stomach of the first pass effect, so that the active ingredients longer, while promoting the body of patients with nitrogen material faster discharge.

Rhubarb aconite soup ${ }^{[14]}$ and rhubarb aconite soup, the prescription from the "Golden Chamber of the main abdominal full of hernia feeding pulse treatment of the tenth" "under the pain, fever, the pulse string, this is known as cold. To lower down the temperature have to use the drug, which is rhubarb aconite soup." The whole side of the attack and play, both cold and heat, both to eliminate the turbid excretion of blood stasis cure its standard, but also warm spleen and kidney rule of the original. Both from the risk of injury and no 
"closed door and keep the invader" the disadvantages. This treatment of cloud poison intrinsic, spleen and kidney weak in Yang commonly used a prescription. The decoction used in this study is composed of rhubarb, weeds, danshen, raw astragalus, poria, alisma, atractylodes macrocephala, Asarum octopus, which rhubarb in the catharsis and discharging turbid, diarrhea catharsis, cooling blood detoxification, cleaning stingy, remove blood stasis and promote blood circulation, to diarrhea for the supplement, for the monarch medicine; system aconite can warm the spleen and kidney, keep warm in the cold, cured is strengthen body resistance and eliminating evil factors, only abdominal pain; Asarum has acrid-warm for ventilation, water resuscitation, remove cold and stop the pain, Alisma, white water and wet water, spleen dampness and dampness, and so on. It can be used as a medicine. The Rhubarb, although bitter cold, but with Asarum, aconite compatibility, then the cold was system and diarrhea, and work still, for the nature of access to the law. Rhubarb, Asarum and aconite three drugs together, with a warm and cold condensate, bitter pass down the effect.

Modern pharmacological studies ${ }^{[15]}$ confirmed that rhubarb can inhibit glomerular mesangial cells and interstitial accumulation and abnormal proliferation, so that the process of glomerular sclerosis delay, while reducing renal interstitial fibrosis; aconite can reduce the methyl bird purine content, improve patients with low calcium symptoms and hyperphosphatemia; Asarum has antihypertensive, anti-inflammatory, anti-allergic effects, but also reduce the excretion of urinary protein in kidney rats. In this study, three drugs used simultaneously, can promote BUN, Cr with the discharge of urine, delay the progress of CRF. The results showed that rhubarb aconite combined with acupoint application in the treatment of CRF patients, can reduce TCM syndrome score, improve the level of renal function indicators, less adverse reactions, thereby delaying the progress of CRF, and effectively alleviate the clinical symptoms of patients.

In summary, CRF patients to rhubarb aconite Asarum combined with acupoint application, efficient and safe, worthy of clinical application.

\section{References}

[1] Wang Zongqian, Yin Li, Zhang Siqing. 937 Cases of Chronic Renal Failure Analysis of Etiology [J]. Chinese Journal of Practical Internal Medicine, 2013, 33 (1): $58-61$

[2] Aimaretti L A, Arze S. Preemptive Renal Transplantation-The Best Treatment Option for Terminal Chronic
Renal Failure [J]. Transplant.Proc., 2016,48 (2): 609611

[3] Shen Hui, Tao Hanhua, Zhang Zhao, et al. Chronic Renal Failure Clinical Research Progress [J]. Liaoning Journal of Traditional Chinese Medicine, 2014, 41 (3): 597-600

[4] Chen Hanzhu. Practical Internal Medicine, the 11th edition [M]. Beijing: Beijing Health Publishing House, 2002: 2364

[5] Zheng Xiaoyu. Traditional Chinese Medicine Clinical Research Guidelines [M]. Beijing: China Pharmaceutical Science and Technology Press, 2002: 163-168

[6] Kim JW, Ha GY, Yong W J. Chronic Renal Failure Induces Cell Death In Rat Hippocampal CA1 Via Upregulation Of Acamkii / NR2A Synaptic Complex And Phosphorylated Glur1-Containing AMPA Receptor Cascades [J]. Kidney Res.Clin.Pract. , 2014, 33 (3): 132-138

[7] Sun Xin, Cong Rijie. Chinese Medicine Treatment of Chronic Renal Failure Research Progress [J]. Chinese Journal of Traditional Chinese Medicine, 2015, 21 (1): $77-78$

[8] Li Tao, Wang Baohe, Yi Chunmei. Chronic Kidney Failure of Traditional Chinese Medicine Research Overview [J]. Chinese Journal of Integrated Traditional Chinese and Western Medicine, 2014, 15 (4): 371-373

[9] Yang Bo, Qiao Yanheng, Zhao Yanru, et al. Exploring TCM Etiology and Pathogenesis of Chronic Renal Failure By Analyzing the Literature of Ten Years [J]. Liaoning Journal of Traditional Chinese Medicine, 2016,43 (1): 58-59

[10] Zeng Wenjun. Chinese Medicine Comprehensive Syndrome Differentiation of Chronic Renal Failure Clinical Study [J]. Asia Pacific Traditional Medicine, 2016, 12 (10): 117-118

[11] Wen Yumin, Zhang Hexuan, Yan Meixuan, et al. Rhubarb and Its Preparation in the Treatment of Chronic Renal Failure in the Overview [J]. Liaoning Journal of Traditional Chinese Medicine, 2015, 42 (10): 20302032

[12] Hu Yaojun, Liu Yanlan. Chinese Medicine Retention Enema Combined with Traditional Chinese Medicine Acupoint Application of Chronic Renal Failure Treatment of the Efficacy of Observation [J]. Hubei Journal of Traditional Chinese Medicine, 2015,37 (1): 46-47 
[13] Qian Shaobing. Chinese Medicine Acupoints Combined With Fuzhenghua Decoction In The Treatment Of 40 Cases Of Chronic Renal Failure Clinical Observation [J]. Hebei Traditional Chinese Medicine, 2015, 37 (2): 177-179

[14] Wang Bing. Different doses of Rhubarb and Radix Aconitum Decoction on TCM Syndrome Integration and Renal Function in Patients with Chronic Renal Failure [J]. Journal of Practical Clinical Medicine, 2012, 16 (17): $34-36$

[15] Liu Yucong. Rhubarb Aconite Soup Enema Treatment of Chronic Renal Failure in Clinical Experience $[J]$. Shenzhen Journal of Integrated Traditional and Western Medicine, 2015, 25 (5): 61-62 